\title{
Helicobacter Pylori infection and Persistent Itching
}

\author{
Rasha Ibrahim Salama ${ }^{1}$, Amira Amin Salem Tawoos ${ }^{2}$, Ehab Darwiesh ${ }^{1}$ \\ ${ }^{1}$ Department of Tropical Medicine, Faculty of Medicine, Zagazig University, Zagazig, \\ Egypt . \\ ${ }^{2}$ Department of Pathology, Faculty of Medicine, Zagazig University, Zagazig, Egypt.
}

Corresponding Author Ehab Darwiesh

Mobile:

+966542603466

E mail: rashasalama1010@gm ail.com

Key words: Helicobacter pylori, Itching, $\operatorname{Ig} E$
Background and study aim: Helicobacter $(H)$ pylori is a worldwide prevalent infection with wide range of gastric and extra-gastric diseases. There is evidence in the literature linking $H$ pylori to many skin diseases. The current study aimed at determining the frequency of $H$ pylori among patients with persistent itching and evaluates impact of $H$ pylori treatment on the course of the disease.

Patients and Methods: Two hundred eighty patients with persistent itching were tested for $H$ pylori antigen in their stool. Patients with and without $H$ pylori antigen positivity were compared. $H$ pylori positive cases were treated by 2 weeks regimens.

Results: One hundred thirty patients (46.4\%) were positive for $H$ pylori stool antigen. Patients with and without $H$ pylori stool antigen positivity were comparable in their demographic and laboratory parameters except for $\mathrm{Ig} \mathrm{E}$ which was more positive among $H$ pylori negative cases. $H$ pylori positive cases had $60 \%, 20 \%$, and $20 \%$ rates of complete resolution, partial resolution and non-resolution of itching after $H$ pylori treatment respectively.

Conclusion: Among patients with persistent itching recruited in the current study, $46.4 \%$ had an evidence of $H$ pylori infection. $H$ pylori positive cases with persistent itching did not have elevated Ig E levels. Out of $130 \mathrm{H}$ pylori positive cases $60 \%$ had complete resolution of itching after treatment of $H$ pylori.

\section{INTRODUCTION}

Helicobacter $(H)$ pylori is the most prevalent infection around the globe with higher prevalence rate in the developing countries. Although this gram negative spiral bacteria inhabits the gastro-duodenal mucosa; it is linked to gastric and many extragastric disease states. The spectrum of gastro-duodenal affection mostly passes through asymptomatic infection. Furthermore, with time the infection evolves from acute and chronic gastritis, peptic ulcer to the most serious hazard; the gastric cancer $[\mathbf{1 , 2}]$.

The extra-gastric effects attributed to $H$ pylori are matters of discussion in the literature. It had been postulated to have an impact on many metabolic, cardiac, neurological, allergic, ocular, haematological, dermatological, and hepatobiliary diseases. However, many of the attributed effects are no more than epidemiological associations [3]. Among the confirmed extra-gastric effects attributed to $H$ pylori infection are iron deficiency anemia, primary immune thrombocytopenia (ITP), and vitamin B12 deficiency which necessitates a test and treat strategy for $H$ pylori infection $[\mathbf{1 , 2 ]}$.

The association between $H$ pylori infection and skin diseases was postulated not only from epidemiological studies, but also from clinical studies [4]. Previous reports documented a possible relation between active $H$ pylori infection and allergic and non-allergic skin diseases $[4,5]$. The aim of the current study is to investigate the frequency of $H$ pylori infection among patients with persistent itching and evaluate the impact of $H$ pylori treatment on the course of the disease. 


\section{PATIENTS AND METHODS}

This was a prospective study conducted over 2 years from March 2019 to February 2021 recruiting patients with persistent itching. All patients were evaluated initially in dermatology clinics before being referred for further evaluation in gastroenterology clinics. Patients with persistent itching were examined for $H$ pylori antigen in their stools after the standard precautions. Patients were divided into 2 groups. Groups I; are patients with persistent itching and positive H. pylori antigen in stool and Groups II; are patients with persistent itching and have negative results for $H$. pylori antigen in stool. Patients of both groups were compared for their demographics, clinical and laboratory data prospectively.

\section{Definitions:}

- Itching or pruritis: Defined in this study as persistent sensation of itching in absence of any identifiable local or systemic causes.

- Response to treatment: is defined as complete or partial disappearance of the intense desire for itching. In $H$ pylori positive group assessment of the response was done at least 4 weeks after finishing the treatment.

- Treatment of $H$ pylori followed the guidelines and vary from one case to another per the circumstances [1]. For treatment naïve patients the triple therapy (PPI, clarithromycin, and amoxicillin) was used, while for patients with recent exposure to or potential risk of resistance to clarithromycin, levofloxacin based quadruple therapy was used [1,2].

\section{Inclusion criteria:}

- Adult patients of 18 years and older

- Patients with persistent itching

\section{Exclusion criteria}

- Patients unwilling to participate

- Patients who received treatment for $H$ pylori or PPI within 4 weeks prior to the commencement of the study.

- Patients with known allergic diseases e.g. allergic asthma

- Patients with known skin diseases

- Patients with known allergy to the proposed anti- $H$ pylori treatments

\section{All patients were subjected to:}

- Full history taking including any history of allergic diseases

- Thorough clinical examination

- $H$ pylori antigen detection in stool. (On Site H. pylori Ag Rapid Test, CTK Biotech, USA). It is a lateral flow chromatographic immunoassay for qualitative assessment of $H$. pylori antigens depending on the use of monoclonal antibodies against $H$. pylori conjugated with colloid gold. Positive cases were marked with two bands of color changes (one test band and one control band).

- Lab work up that included: ALT, AST, CBC, $\mathrm{TSH}$, serum bilirubin, creatinine, serum Ig E, CRP, ESR

- $H$ pylori positive cases were treated by 2 weeks course

\section{Statistical analysis}

Primary end point of the current study was determination of $H$ pylori prevalence among patients with persistent itching

Secondary end point of the current study was determination the response to $H$ pylori treatment among positive cases with persistent itching.

Data were analyzed using SPSS version 20. Data were expressed as number and percentage for qualitative variables and mean \pm standard deviation for quantitative ones. $\mathrm{P}$ value of $<0.05$ indicates significant results. Comparison between the two groups was done using $t$-test and Chi-square test.

\section{RESULTS}

\section{Patient demographics}

This study included 280 consecutive patients with persistent itching and according to their positivity $(\mathrm{n}=130)$ or negativity $(\mathrm{n}=150)$ to $H$ pylori stool antigen were classified as group I and II respectively. The prevalence of $H$ pylori among patients with persistent itching is $46.4 \%$ (130/280). The age and gender were comparable in both groups (Table 1).

\section{Laboratory data of both groups}

When both groups were compared regarding the common causes of persistent itching, it was clear that liver biochemical profile and thyroid function were comparable between the 2 groups (Table 2). The acute phase reactants CRP and ESR and CBC were also comparable between the 
2 groups. However, the $H$ pylori negative group had higher frequency (73.4\%) of IgE positivity in comparison to none $(0 \%)$ among $H$ pylori positive group $(\mathrm{P}<0.001)$.

\section{Response to H Pylori treatment}

In this study all $H$ pylori positive cases were treated for 2 weeks duration. Treatment was individualized case by case according to the situation e.g. previous treatment failure........etc. The response to treatment was evaluated 4 weeks after completion of the treatment course. Out of the $H$ pylori positive patients $60 \% \quad(n=78)$ had complete resolution of symptoms, while $20 \%$ $(n=26)$ had partial response in contrast to $20 \%$ $(\mathrm{n}=26)$ who did not improve (Figure 1). When compared to the $H$ pylori negative group who showed no response to anti-histamines.

Table (1): Demographic data distribution between studied groups.

\begin{tabular}{|l|c|c|c|}
\hline & $\begin{array}{c}\text { Group I } \\
(\mathbf{n = 1 3 0})\end{array}$ & $\begin{array}{c}\text { Group II } \\
(\mathbf{n = 1 5 0})\end{array}$ & P \\
\hline Age (years) & $47.26 \pm 11.74$ & $45.28 \pm 10.88$ & 0.095 \\
\hline Sex & $6247.6 \%$ & $6946.0 \%$ & 0.77 \\
& $6852.4 \%$ & $8154.0 \%$ & \\
\hline Male & 68 Female & 68 &
\end{tabular}

No significant difference or association between groups.

Table (2): Lab characteristics of both groups.

\begin{tabular}{|c|c|c|c|}
\hline & $\begin{array}{l}\text { Group I } \\
(\mathbf{n}=130)\end{array}$ & $\begin{array}{c}\text { Group II } \\
(\mathbf{n}=\mathbf{1 5 0})\end{array}$ & $\mathbf{P}$ \\
\hline $\begin{array}{c}\text { Thyroid function } \\
\text { Normal } \\
\text { Abnormal }\end{array}$ & $\begin{array}{ll}112 & 86.2 \% \\
18 & 13.8 \%\end{array}$ & $\begin{array}{cc}120 & 80.0 \% \\
30 & 20.0 \%\end{array}$ & 0.174 \\
\hline SGOT (IU/L) & $29.63 \pm 9.36$ & $32.57 \pm 10.87$ & 0.211 \\
\hline SGPT (IU/L) & $31.58 \pm 7.41$ & $33.63 \pm 8.88$ & 0.136 \\
\hline Total bilirubin (mg/dl) & $1.21 \pm 0.22$ & $1.27 \pm 0.35$ & 0.0856 \\
\hline \begin{tabular}{|ll} 
CRP & \\
& Negative \\
& Positive \\
\end{tabular} & $\begin{array}{cc}130 & 100.0 \% \\
0 & 0.0 \% \\
\end{array}$ & $\begin{array}{cl}144 & 96.0 \% \\
6 & 4.0 \% \\
\end{array}$ & 0.058 \\
\hline \begin{tabular}{|ll}
$\mathbf{I g} \mathbf{E}$ & \\
& $\begin{array}{l}\text { Negative } \\
\text { Positive }\end{array}$ \\
\end{tabular} & $\begin{array}{rl}130 & 100.0 \% \\
0 & 0.0 \% \\
\end{array}$ & $\begin{array}{cc}110 & 73.4 \% \\
40 & 26.6 \% \\
\end{array}$ & $0.00 * *$ \\
\hline Elevated ESR & $0 \quad 0.0 \%$ & $0 \quad 0.0 \%$ & 1.0 \\
\hline Hemoglobin (gm/dl) & $12.25 \pm 3.08$ & $11.88 \pm 2.85$ & 0.35 \\
\hline WBCs x10 ${ }^{3}$ & $7.56 \pm 1.85$ & $6.99 \pm 2.12$ & 0.33 \\
\hline${\mathrm{PLT} \times 10^{3}}$ & $187.63 \pm 60.63$ & $179.36 \pm 58.36$ & 0.23 \\
\hline
\end{tabular}

*** Highly significant 


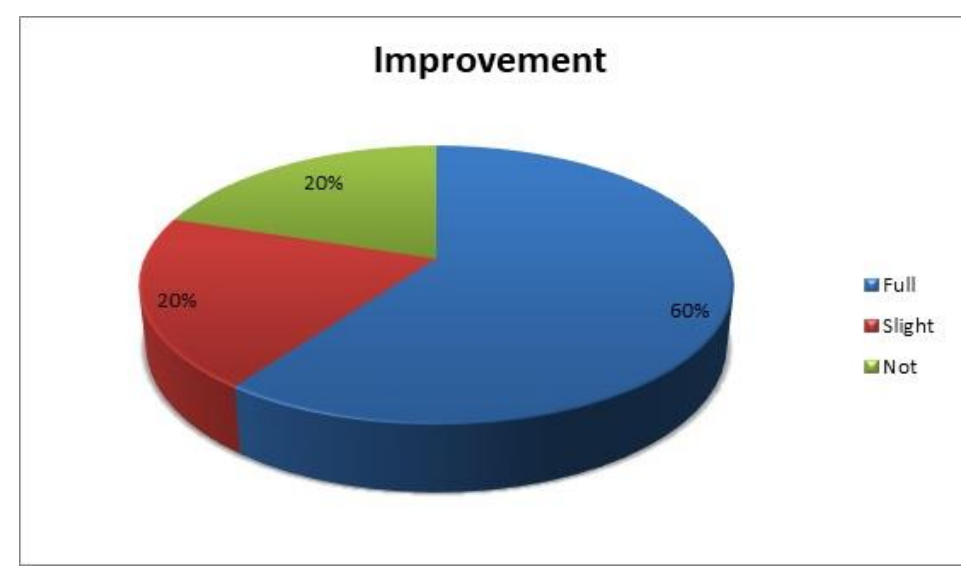

Figure (1): Patterns of response among $H$ pylori positive patients.

\section{DISCUSSION}

$H$ pylori have been linked to many skin diseases in many epidemiological studies as well as clinical ones. The link of skin diseases mainly pruritic skin diseases among $H$ pylori patients have been attributed to many mechanisms. The most widely accepted is autoimmune one. It seems that following the prolonged infection with $H$ pylori, an interaction with the host immune system do occur. $H$ pylori trigger antigens that cross-react with $\mathrm{T}$ cells and provoke autoantibodies resulting in loss of tolerance to self-antigens in the skin [6]. The association of $\mathrm{H}$ pylori to allergic diseases have been questioned and some reports postulated protective role of $H$ pylori against allergy with documentation of an inverse relationship between prevalence of $H$ pylori and allergic diseases [7]. The lack of Ig E detection among $H$ pylori patients with pruritis in this study may favor this assumption

There is growing practice behavior among dermatologists in our community to investigate cases of persistent itching for $\mathrm{H}$ pylori. Although this is not supported by any current clinical practice guidelines [1,2], however it may be justified by the growing evidence in the literature linking $H$ pylori to a variety of skin disorders [5,8]. The high prevalence of $H$ pylori among patients with persistent itching (46.4\%) reported in the current study is another support to this trend. In fact, this high prevalence is in agreement with Kandyil et al. [9], who reported H pylori prevalence rate of $80 \%$ (8/10 patients) among patients with refractory pruritus and also with Saleh et al. [10], who reported a rate of $63.4 \%$ (50/63) among patients with rosacea.
In the current study there were no significant differences between both groups as regard the age and sex. This is in agreement with Marae et al., who reported similar results for patients having palm and sole of psoriasis with and without $H$ pylori [11].

The common causes of itching usually seen in our community e.g. liver diseases and thyroid dysfunction were ruled out. This favors the hypothesis that $H$ pylori is presumably a direct cause of itching and the link is not just an epidemiological association. The lack of $\mathrm{Ig} \mathrm{E}$ positivity among our $H$ pylori positive cohort $(0 \%, \mathrm{P}<0.001)$ favors the possibility that a direct non-allergic mechanism stands behind. Although we did not found studies focusing $H$ pylori and skin diseases targeting Ig $\mathrm{E}$ measurement, one study among children showed high prevalence of food specific Ig E levels among children with $H$ pylori infection [12] probably this finding is not applicable for skin diseases.

The results of the current study are in agreement with the study of Kandyil et al. [9], who reported a possible relationship between refractory pruritus and active $H$ pylori infection which was confirmed among 8 out 10 patients focused. The terms of response after $H$ pylori treatment reported by Kandyil et al., were $62.5 \%(5 / 8)$ complete relief, $25 \%(2 / 8)$ temporary relief of pruritus, while the remaining $12.5 \%$ (1/8) did not respond. These figures were not different from the $60 \%, 20 \%$, and $20 \%$ reported respectively in the current study. The clinical terms of response defined in the current study and in the study of Kandyil et al., are usually the target of practitioners and patients as well rather than bacteriological terms of $H$ pylori eradication and this would explain why confirmation of 
eradication was not an endpoint of both the current study nor in the study of Kandyil et al.

We believe that both the high frequency of $H$ pylori among patients with persistent itching and the high $(60 \%)$ itching relief rate reported in the current study would alarm both dermatologists and gastroenterologists in our developing countries to consider $H$ pylori in the differential diagnosis. However, we believe it is an advice rather than a recommendation based on the available evidence.

The current study had some limitations. First, the lack of $H$ pylori detection by more accurate methods of diagnosis e.g. urea breath test (UBT), upper endoscopy and histopathology. Because we performed $H$ pylori stool antigen detection under the standard precautions and hence its sensitivity would be as high as UBT, similar to what we reported in earlier studies [13]. Furthermore, in our clinical practice stool antigen is frequently used due to cost and availability issues. Second, is the lack of $H$ pylori eradication confirmation after treatment. Previous studies [9-11] did not target eradication as an end point.

In conclusion, among patients with persistent itching focused in the current study, $46.4 \%$ had an evidence of $H$ pylori infection. $H$ pylori positive cases with persistent itching did not have elevated Ig E levels. Out of $130 \mathrm{H}$ pylori positive cases $60 \%$ had complete resolution of itching after treatment of $\mathrm{H}$ pylori.

\section{Funding: None.}

Conflict of interest: None.

\section{Ethical consideration:}

All patients signed an informed consent to participate in the study and to perform the relevant investigations after explanation of all steps. The current study was carried in concordance with sound medical practice and in line the ethics raised in the declaration of Helsinki.

\section{ACKNOWLEDGMENT}

- The authors would thank all colleagues who helped to conduct this study.

\section{REFERENCES}

1. Malfertheiner P, Megraud F, O'Morain CA, Atherton J, Axon AT, Bazzoli F, et al.
Management of Helicobacter pylori infection-the Maastricht IV/ Florence Consensus Report. Gut. 2012 May; 61(5):646-64.

2. Alboraie M, Elhossary W, Aly OA, Abbas B, Abdelsalam L, Ghaith D, et al. Egyptian recommendations for management of Helicobacter pylori infection: 2018 report. Arab J Gastroenterol. 2019 Sep; 20(3):175-179.

3. Gravina AG, Zagari RM, De Musis C, Romano L, Loguercio C, Romano M. Helicobacter pylori and extragastric diseases: A review. World $J$ Gastroenterol. 2018 Aug 7; 24(29):3204-3221.

4. Yorulmaz A, Kulcu SC. Helicobacter pylori and inflammatory skin diseases. World J Dermatol 2015; 4(3): 120-128.

5. Guarneri C, Ceccarelli M, Rinaldi L, Cacopardo B, Nunnari G, Guarneri F. Helicobacter pylori and skin disorders: a comprehensive review of the available literature. Eur Rev Med Pharmacol Sci. 2020; 24(23):12267-12287.

6. Magen E, Delgado JS. Helicobacter pylori and skin autoimmune diseases. World $J$ Gastroenterol. 2014 Feb 14;20(6):1510-6.

7. Daugule I, Zavoronkova J, Santare D. Helicobacter pylori and allergy: Update of research. World J Methodol. 2015; 5(4):203-11.

8. Yang, X. Relationship between Helicobacter pylori and Rosacea: review and discussion. $B M C$ Infect Dis 2018;18:318.

9. Kandyil R, Satya NS, Swerlick RA. Chronic pruritus associated with Helicobacter pylori. $J$ Cutan Med Surg. 2002;6(2):103-8.

10. Saleh P, Naghavi-Behzad M, Babapour S, Piri R. The Association Between Helicobacter pylori Infection and Rosacea, Arch Clin Infect Dis. 2018; 13(1):e57740

11. Marae AH, Shehata WA, Azmy R, Abousaeida AM. Helicobacter pylori infection in the palm and sole of psoriatic patients. Menoufia Med J 2021;34:71-5

12. Kolho KL, Haapaniemi A, Haahtela T, Rautelin $\mathrm{H}$. Helicobacter pylori and specific immunoglobulin $\mathrm{E}$ antibodies to food allergens in children. $J$ Pediatr Gastroenterol Nutr. 2005;40(2):180-3.

13. Emara MH, Salama RI, Salem AA. Demographic, Endoscopic and Histopathologic Features Among Stool H. pylori Positive and Stool H. pylori Negative Patients With Dyspepsia. Gastroenterology Res. 2017; 10(5): 305-310. 